\title{
Association between Repeated Sprint Tests Integrated In the Physical Education Program and Anxiety in Adolescent Students
}

\author{
Souhail Hermassi ${ }^{1,2}$, Naila Bali ${ }^{1,2}$, Karim Chamari ${ }^{3}$ \\ 1 (Tunisian Research Laboratory « Sport Performance Optimization », Tunis, Tunisia) \\ 2 \\ 3 \\ 2 (High Institute of Sport and Physical Education, Ksar Saîd, Tunis, Tunisia) \\ (Athlete Health and Performance Research Centre, ASPETAR, Qatar Orthopaedic and Sports Medicine \\ Hospital, Qatar)
}

\begin{abstract}
The aim of this study was to show the association between anxiety and the repeated sprint test in adolescent students.

We worked on the central notion of anxiety, 20 students $(17.1 \pm 0.4$ years) agreed to submit to the study by answering questionnaires based on the anixiété. All participants were tested for RSA test $(6 \times(15+15 \mathrm{~m}))$ interspersed with a recovery time of 25 seconds. This test allowed us to calculate and measure the total time $\left(R S A_{T T}\right)$, average time $\left(R S A_{\text {Mean }}\right)$ the fatigue index $\left(R S A_{d e c}\right)$ and the best time $\left(R S A_{\text {best }}\right)$. Statistical analysis revealed a very significant correlation between anxiety and $R_{S A}(r=-0.76, p<0.01)$. Similarly the $R S A_{\text {Mean }}$ and $R_{S A}$ pic is significantly correlated with anxiety $(r=0.65, p<0.01 ;(r=0.78, p<0.01$ respectevely). But no correlation was observed between anxiety and $R S A_{I F}$. The performance of RSA test strongly correlated with anxiety in adolescent students, appear to be useful for evaluating the psychological status of students and may thus allow a revaluation of self and lead a renewed motivation. We can conclude that the absorption of inactivity students requires efforts both pedagogical, didactic and in terms of teacher intervention.
\end{abstract}

Keywords: intermittent test, stress, psychological test, students.

\section{Introduction}

The didactic action places the teacher at the intersection of theory and practice. The focus on various domains (physiological, psychological, or educational) should lead to the design of a coherent content, responsive and accessible to the population. However, during the construction of educational content, the teacher sets goals that are consistent with the level of students but also with the internal logic of the activity (Morth, 1998). These objectives are based on the "fundamentals" of the activity to which they refer.

Learning and teaching is a complex and multidimensional phenomenon and this phenomenon was often due to educational content, yet effective means exist to fight against the lack of investment and engine student engagement. The influence of emotional states on activity and sports performance is a major research topic in sport psychology (Patmore, 1986). Intuitively, it states that this influence is complex if stress can be beneficial to some extent, allowing the subject to mobilize beyond a certain limit, it has instead debilitating effects on performance. Many also agree to admit that one of the key aspects of the expertise of champion is being able to cope with stress and anxiety (Patmore, 1986, Jones \& Hardy, 1989).

In the context of cognitive theory, we can say that anxiety is a response of the body, faced with environmental demands. In the first approach, we can say that anxiety is manifested by the development of negative affect, feelings of apprehension and tension associated with a high level of activation of the body (Martens, Vealey \& Burton, 1990). It is therefore a complex answer, combining cognitive and somatic dimensions.

Recent work in the design a cognitive perspective: anxiety is dependent on intermediate variables represented. Thus, according to Spielberger (1972), state anxiety is related to the perceived threat (threat), that is to say, the evaluation of the current situation as dangerous, physically or psychologically. McGrath (1970) proposes a model of the same type, in which the emotional response arises from the perception of a difference (imbalance) between the difficulty of the task and response capabilities. This response is also dependent on the importance for the subject's success or failure: the perception of a discrepancy between subjective difficulty and skill has a stressful character if achieving the result is a real challenge for the subject. There is a similar logic in a recent book by Martens, Vealey and Burton (1990) devoted to anxiety in sports. The authors believe that the perception of the threat depends on two independent representations: the perceived importance of the outcome, and the perceived uncertainty of the result. 
This perception of emotion as "a state emotionally negative" often incompatible with the production of a high-level performance (Weinberg \& Gould, 1985) has long been unanimous in the literature before being gradually challenged by several studies initiated in the field of academic tasks (Wine, 1980) and sport (Hardy, 1990, Jones 1991, Smith 1996). The authors propose an alternating positive and negative effect of anxiety on performance (Smith, 1996) or the possibility of a favourable (facilitator) or unfavourable interpretation (disturbance) symptoms associated with anxiety. The latter then would own, under certain conditions, potential benefits and facilitating performance (Alpert \& Haber, 1960).

Therefore, recent research in the field of emotions (Lazarus, 2000; Skinner \& Brewer, 2002) equate to more anxiety and a disagreeable unpleasant emotion rather than to a negative emotion. Generally, anxiety is defined as the unpleasant side of emotions. The anxiety is found in everyone, from the youngest to the oldest, but so unique to each specific to each age and culture-specific. But as it is the situation in adolescent athletes?

To our knowledge, there is no scientific reference that examines the relationship between physical stress motion (repeated intermittent-sprint) based to educational content and psychological factors (anxiety) in adolescent students. Our study aims to investigate the relationship the degrees of anxiety and practice of repeated shuttle sprints in adolescent students?

\section{1-Subjects}

\section{Methods}

To accomplish this research, we carried out an experimental study on a population of 20 males students belonging to a secondary school, age $17.1 \pm 0.4$ years. This secondary school was located in the centre of Tunis. These are subjects who practice sports daily at 2 times a week with workouts 2.00 hours on average.

\section{2-Experimental protocols: \\ Repeated sprint ability (RSSA) test:}

Before the test, players completed a warm-up consisting of 10 minutes of jogging supplemented with an additional three to five single $15-\mathrm{m}$ shuttle sprints with two minutes of passive recovery. No static stretching was allowed before the RSSA protocol. An additional 3 min rest was given before players undertook the RSA test protocol. The test consisted of 6 repetitions of maximal $(6 \times(15+15 \mathrm{~m}))$ shuttle sprints with $180^{\circ}$ turns ( $\sim 6$ seconds) departing every 20 s (Buchheit, 2009; Spencer, 2012). During the $\sim 15$-s recovery between sprints, subjects were required to perform an active recovery (brisk walk back to the starting line). Three seconds before starting each sprint, the subjects were asked to assume the start position and await the start signal. Two sets of timing gates (Microgate Srl; Italy) were used, working in opposite directions, to allow subjects to start the next run from the same end at which they had finished the preceding sprint. Three scores were calculated for each RSA test; best sprint time in a single trial $\left(\mathrm{RSA}_{\text {best }}\right)$, total sprint time $\left(\mathrm{RSA}_{\mathrm{TT}}\right)$, average time $\left(\mathrm{RSA}_{\text {Mean }}\right)$ and fatigue $\left(\mathrm{RSA}_{\mathrm{dec}}\right)$, calculated using the percentage decrement method: $100-($ Total time / ideal time $\times 100)$; Where the ideal time $=6 \times$ RSA best (Spencer, 2012).

\section{3-Anxiety Questionnaire-State (CSAI-2):}

Developed by Spielberger in 1966, evaluates feelings of apprehension, tension, nervousness and worry that the subject feels when the anxiety-producing situation or the situation of competition. This questionnaire is an indicator of transient changes of the anxiety caused by aversive or therapeutic situations. In athletes about the competition can be anxiety-generating situations. Anxiety Questionnaire - State (Competitive State Anxiety Inventory, CSAI-2) was developed and validated by Martens, Vealey \& Burton in 1990.

\section{4-The statistical procedures:}

Descriptive statistics were calculated as mean \pm standard deviation (SD). Before using parametric tests, the assumption of normality was verified using the Shapiro-Wilk test. Pearson's product moment correlations and linear regression analysis were used to examine the relationships between RSA performance and anxiety. The level of significance for all tests was set at 0.05. All analyses were performed using SPSS v 13 (SPSS In., Chicago, IL, USA).

\section{Results}

The scores obtained of RSA test performances are shown in Table 1. The correlation between the indices of RSA test and anxiety are shown in Table 2. Statistical analysis revealed a very significant correlation between anxiety and RSATT $(r=-0.76, p<0.01)$. Similarly the $\mathrm{RSA}_{\text {Mean }}$ and $\mathrm{RSA}_{\text {best }}$ is significantly correlated with anxiety $(\mathrm{r}=0.65, \mathrm{p}<0.01 ;(\mathrm{r}=0.78, \mathrm{p}<0.01$ respectively). But no correlation was observed between anxiety and RSAIF. 
Table 1 : Performance measured parameters of RSA test

\begin{tabular}{cccccc}
\hline & $\begin{array}{c}\mathrm{RSA}_{\text {TT }} \\
(\mathrm{s})\end{array}$ & $\begin{array}{c}\mathrm{RSA}_{\text {Mean }} \\
(\mathrm{s})\end{array}$ & $\begin{array}{c}\mathrm{RSA}_{\text {Best }} \\
(\mathrm{s})\end{array}$ & $\begin{array}{c}\mathrm{RSA}_{\text {Dec }} \\
(\mathrm{s})\end{array}$ & Anxiety \\
\hline Moyenne & 48.25 & 8.13 & 8.32 & 3.90 & 40.19 \\
$\pm \mathrm{SD}$ & 2.7 & 0.45 & 0.53 & 0.08 & 10.86 \\
\hline
\end{tabular}

Table $2:$ The correlation between the performance of RSA test and anxiety

\begin{tabular}{ccccc}
\hline & $\mathrm{RSA}_{\mathrm{TT}}(\mathrm{s})$ & $\mathrm{RSA}_{\text {Mean }}(\mathrm{s})$ & $\mathrm{RSA}_{\text {Best }}(\mathrm{s})$ & $\mathrm{RSA}_{\text {Dec }}(\mathrm{s})$ \\
\hline Score Anxiété Pre & $0.76^{* *}$ & $0.65^{*}$ & $0.78^{* *}$ & 0.10 \\
\hline \multicolumn{4}{c}{$\mathrm{p}<0.01 ; \mathrm{p}<0.05$}
\end{tabular}

\section{Discussion}

The results shows that the relationship between the performances of RSA test was a very advanced psychological state as manifestations of emotion appear in the presence of a given real situation, anxiety can be considered a pointless fear, a feeling of insecurity. It is triggered by different causes, future or imaginary situations experienced as a threat, or at least something difficult to overcome, can be linked to intrapsychic conflicts or in connection with the outside world, anticipation of an action or risk considered as such. "(Spielberger,1966). On the psychic level, anxiety is still felt so painful, it differs again from emotion. Anxiety is a negative emotional state that is accompanied by tension, anxiety, apprehension, associated with activation of the body (Ntoumanis \& Biddle, 2000). It thus has a cognitive component characterized by subjective sensations of apprehension and tension induced by a risk of failure and a somatic component corresponding to physiological events collected during the anxiety-producing situation. The EPS sessions generally should allow students to use their energy resources through the intensity and duration of the forces induced by the tasks that the teacher has the knowledge that the child of this age recovers quickly. The way to get an idea of the limits of the intensity of effort that each student is able to provide is to make him a stress test to know its maximum aerobic speed (without oxygen debt) realize that should incorporate as a benchmark running speed not to exceed whatever programmed by the teacher in which the student has to run long activity. This test will allow the teachers evaluate student performance to make intense efforts on limited short distances, more variety of tasks proposed race speed will allow him to find interest in racing games besides allowing them to place its performance in space and time with respect to itself and with respect to the other. Obstacle courses, relays and flat made parallel in the same session will offer a wide and diversified range challenging tasks. The principle of the systematic measurement of space and time is essential for the consolidation cycle for the student to construct concrete representations of measuring the amplitude and frequency.

The results also show that the more confident students tend to perceive situations as identified problems and respond with a strategy to change the problem. The results suggest that self-esteem influences the interpretation of the defeat and the students have a lot of confidence in themselves to protect their personal effectiveness in a much more efficient and use more coping strategies than the less confident students in their abilities. For this, the orientation of the logic of the EPS can be done through the performance: "out of the logic of performance that is still too much room for more students to take the notion of pleasure. So they will want to practice later "" reintroduce the investment share of the student back to the old discipline as "values" consideration of participation (investment, stress, courage , motivation, desire ...) to APS in the school newsletter, "" the evaluation criteria should be reviewed to better take into account the specificity of students EPS "should that" transdisciplinarily be increased; "to" make EPS of a key link in a cross-disciplinary approach, "" Work more in development and physical capacity, hygiene and respect for the body, pleasure and sharing it with others, fraternity and type of relationship it engenders "" a larger share of "citizenship" in the ratings exams, many of which seem fanciful. "It would also "allow the EPS to play its role in school integration of pupils" and allow "the integration of students suffering from physical weakness".

\section{Conclusion}

The results suggest that Anxiety influences the interpretation of the defeat and the students have a lot of confidence in themselves to protect their personal effectiveness in a much more efficient and use more coping strategies than the less confident students in their abilities. For this, the orientation of the logic of the physical program school can be done through the performance: "out of the logic of performance that is still too much room for more students to take the notion of pleasure. So they will want to practice later "" reintroduce the investment share of the student back to the old discipline as "values" consideration of participation (investment, 
stress, courage , motivation, desire ...) in the school newsletter, "" the evaluation criteria should be reviewed to better take into account the specificity of students "should that" transdisciplinarily be increased; "to" make physical program school of a key link in a cross-disciplinary approach, "" Work more in development and physical capacity, hygiene and respect for the body, pleasure and sharing it with others, fraternity and type of relationship it engenders "" a larger share of "citizenship" in the ratings exams, many of which seem fanciful. "It would also "allow the physical program school to play its role in school integration of pupils" and allow "the integration of students suffering from physical weakness".

\section{References}

[1]. Alpert, R., \& Haber, R. N. (1960). Anxiety in academic achievement situations. Journal of Abnormal and Social Psychology, 61, 207-215.

[2]. Bishop, D \& Castagna, C (2002). La scienza della « repeated sprint ability » teknosport $\mathrm{n}^{\circ} 24$.

[3]. Buchheit M, Lepretre PM, Behaegel AL, Millet GP, Cuvelier G, \& Ahmaidi S. (2009).Cardiorespiratory responses during running and sport-specific exercises in handball players. J Sci Med Sport 12: 399-405,

[4]. Gould, D., \& Weinberg, R. S. (1985). Sources of worry in successful and less successful intercollegiate wrestlers. Journal of Sport Behavior, 8(2), 115-127.

[5]. Hardy, L (1990). A catastrophe model of anxiety and performance. In J. G. Jones \& L. Hardy (Eds.). Stress and performance in sport (pp. 81-106). Chichester, England: Wiley.

[6]. Jones, J. G. (1995). More than just a game : Research developments and issues in competitive anxiety in sport. British Journal of Psychology, 86, 449-478.

[7]. Jones, J.G. \& Hardy, L. (1989). Stress and cognitive functionning in sport. Journal of Sport Science, 7, 41-63.

[8]. Lazarus, R. S. (2000). How emotions influence performance in competitive sports. The Sport Psychologist, 14, $229-252$.

[9]. McGrath J. E. (1970). Social and psychological factors in stress. New York: Holt Rinehart and Winston.

[10]. Martens, R., Vealey R. S., \& Burton D. (Eds.) (1990). Competitive Anxiety in Sport. Champaign, IL: Human Kinetics Publishers.

[11]. Patmore, A. (1986). Sportsmen under Stress. London: Stanley Paul.

[12]. Skinner, N., \& Brewer, N. (2002). The dynamics of threat and challenge appraisals prior to stressful achievements events. Journal of Personality and Social Psychology, 83(3), 678-692

[13]. Smith, R. E. (1996). Performance anxiety, cognitive interference, and concentration enhancement strategies in sport. In I. G. Sarason, G. R. Pierce \& B. R. Sarason (Eds.), Cognitive interference: Theories, methods and findings (pp. 261-283). Lauwrence Erlbaum Associates.

[14]. Spielberger, C. D. (1972). Anxiety as an emotional state. In C. D. Spielberger (Ed.), Anxiety: Current trends in theory and research (Vol. 1, pp. 23-49). New York: Academic Press.

[15]. Wine, J. D. (1980). Cognitive-attentional theory of test anxiety. In I. G. Sarason (Ed.), Test anxiety: Theory, research and applications (pp. 349-385). Hillsdale, NJ: Erlbaum. 